\title{
Mouse Models in Cardiology Research
}

\author{
Aasakiran Madamanchi*, BSc
}

\section{INTRODUCTION}

A variety of interacting genetic and environmental factors contribute to the onset of cardiovascular disease. Diet, exercise, other lifestyle changes, and hereditary factors are all associated with heart disease. Because of its complex etiology, however, heart disease is difficult to treat. The recombinant DNA revolution in the $1970 \mathrm{~s}$ provided the technology for biomedical researchers to investigate the genetic and molecular basis for many complex human diseases such as heart disease. Molecular genetic techniques enable scientists to create animal models that mimic various aspects of cardiovascular disease. Studying the effect of highly specific genetically engineered changes offers insight into the genetic and molecular mechanisms that underlie the onset of heart disease. Recently, the mouse has emerged as the most prevalent experimental model for cardiovascular research. Formerly, larger mammals such as pigs and rabbits had been the preferred experimental animals. Many researchers perceived the differences in cardiac size and morphology to be so large as to preclude the use of mice as a relevant experimental model for human cardiovascular disease. As the comparative ease in applying recombinant DNA technology in mice (relative to other species) became apparent, the popularity of murine experimental models grew. The advantages of short gestation periods, costeffective maintenance, and a well-characterized genome make the mouse the ideal experimental model (1). Animal models are useful not only to elucidate the molecular mechanisms that underlie heart disease, but also to test experimental therapies. Recently, the miniaturization of technologies for measuring cardiac endpoints has vastly increased the variety of

\footnotetext{
* To whom correspondence should be addressed: Asakiran Madamanchi, Box 97787, Durham, NC 27708, tel. (919) 613-2582 email: am60@duke.edu
}

cardiovascular traits that can be modeled. In 1992, a mouse deficient in apolipoprotein $\mathrm{E}$, the apoE -/mouse, was generated as the first mouse-line with a stable genetic background that developed spontaneous arterial lesions and coronary artery occlusion (2,3). Since that time, the size and scope of cardiovascular research on genetically engineered mouse models has grown prodigiously. The purpose of this review is to survey some important murine models and the available procedures for measuring cardiac endpoints as well as to explore new directions for the use of murine models in cardiovascular research.

\section{PROMINENT MODELS}

\section{Atherosclerosis}

Atherosclerosis, the progressive hardening of the arteries that leads to their blockage and rupture, is a very complex disease that progresses steadily for many years before clinical symptoms can be seen. The pathophysiology of atherosclerosis is initiated by the development of streaks of fatty material on the interior of the vessel wall. Damage to vascular endothelium by circulating mediators and physical forces leads to endothelial dysfunction and precedes fatty-streak formation. Oxidized low-density lipoprotein (Ox-LDL) is one of the mediators of endothelial damage. The transport of Ox-LDL across the endothelium into the arterial wall is necessary for the formation of fatty streaks and their progression to advanced atherosclerotic lesions (4). As these lesions, which are covered by proliferating smooth muscle cells, grow in size, the vessel lumen is narrowed, resulting in disturbed blood flow and hemodynamic forces that ultimately lead to fibrous plaques. In acute coronary syndromes, fibrous plaques rupture and initiate thrombus formation that results in vessel occlusion (5).

There is currently no existent mouse strain that generates spontaneous arterial lesions while on a 
normal diet. Of the inbred strains, C57BL/6 is the most susceptible to atherosclerosis and it is therefore the strain of choice to be genetically manipulated to produce atherosclerotic models (6). Even in this strain, however, diet-induced atherosclerosis is restricted to small-lesion growth in the aortic root. In the apoE knockout mouse, gene-targeting techniques were used to delete the gene that coded for apolipoprotein E, a lipoprotein surface component and ligand necessary for interaction with a number of lipoprotein-related receptors (3). All apoE -/- mice develop hyperlipidemia and atherosclerosis on both normal and high-fat diets, although the severity of the phenotype is significantly strain-dependent. This has been the most studied and best characterized model for atherosclerosis. ApoE -/mice often serve as a background for additional genetic manipulation and this approach allows for the assessment of the role of individual genes in the progression of atherosclerosis or their function under atherosclerotic conditions. Unfortunately, the apoE -/background is not a wholly accurate model for human atherosclerosis; the lipoprotein profiles of apoE -/- mice differ significantly from those of typical human patients and the absence of apoE is a rare condition in humans (8).

Another atherosclerosis model, the LDL receptor -/mouse, is one of the most common backgrounds used for compound genetic manipulation. The deficiency in LDL receptors results in a less severe form of hypercholesterolemia (9). On high-fat diets, animals develop hypercholesterolemia and atherosclerosis manifests. This mouse model is particularly susceptible to dietary influence; a small change in diet is sufficient to reveal the atherosclerotic phenotype. Interestingly, LDL receptor deficiency is a phenotype that is found in humans (8). In addition to the gene-targeted knockout models, there are a number of transgenic models for atherosclerosis. Most transgenic models involve the insertion of either human or mutant forms of apolipoprotein E, such as apoE2 or apoE3-Leiden (7). A number of human isoforms of apoE have been expressed in mice to isolate the isoforms' influence on the development of atherosclerosis. When given a highfat diet, mutant and human transgenic apoE mouse strains typically experience moderate hypercholesterolemia and produce atherosclerotic lesions (3). The third type of mouse model for atherosclerosis is based on a gene-replacement method that is known as a 'knock-in'. In contrast to transgenic mice, where the gene of choice can express from a number of copies in various chromosomal locations, in knock-in mice, the endogenous allele is replaced by a variant of the gene in the same location in the genome. This enables the same tissue-specific expression of the variant as that of the endogenous gene (6). Knock-in mice expressing human apoE2 exhibit a plasmalipoprotein protein profile similar to that of type III hyperlipidemic subjects; they are defective in clearing migrating VLDL (very-low-density lipoprotein) particles and develop atherosclerotic lesions even on a regular diet (10).

\section{Vascular Remodeling}

Angioplasty is a medical procedure in which a balloon is used to open coronary arteries narrowed or blocked by the progression of atherosclerosis. Angioplasty is the most common treatment for coronary artery disease; it is less invasive and has a shorter recovery period than bypass surgery (11). The most common complication of angioplasty is the long-term risk of restenosis, or reclosure of the treated artery, a form of vascular remodeling. Restenosis becomes problematic with this procedure because of the arterial injury that is often inflicted during balloon angioplasty that frequently results in chronic narrowing of the lumen due to constrictive remodeling of the vessel wall. Recently, to prevent restenosis, the use of drug-eluting stents have gained wider acceptance in angioplasty. These stents are small, self-expanding stainless-steel mesh tubes that prevent reclosure of the blood vessel by the slow release of antimitotic agents (12).

Several murine injury models have been developed to study the molecular basis of vascular remodeling in response to arterial injury. In mechanical injury models, a nylon wire is used to mechanically injure the murine carotid artery (13). This approach allows for the study of the molecular basis of the smooth muscle cell response to arterial injury. More specifically, this model focuses on the consequences of endothelial denudation and medial injury in the absence of complicating factors such as thrombosis and inflammation. This method produces a model that is particularly relevant to balloon angioplasty in humans and allows preclinical testing of new therapies. Another widespread approach is the perivascular electric injury model in which an electric current is sent through the wall of carotid arteries using an electromicrocoagulator (11). The current induces a severe injury destroying all the cells along the vessel wall. The massive and total cellular death along the site of the injury causes vascular healing to begin at the uninjured sites along the edges of the wound. New endothelial and smooth muscle cells migrate over the injury site. This rapid smooth muscle cell migration and reendothelialization makes the perivascular injury model useful for studying the molecular basis of smooth muscle cell migration during conditions of thrombosis or inflammation. Traditionally, murine injury model experiments have been found to be highly background- 
dependent. Unfortunately, these experiments have been performed on only a limited number of transgenic mouse backgrounds. Local gene transfer in murine arteries has thus far proved unsuccessful because of technical difficulties associated with size constraints, however, systemic gene-transfer strategies appear to show promise. The use of transgenic mice in injury experiments continue to be important as the molecular mechanisms in vascular remodeling are likely to be quite distinct in diseased arteries.

\section{Hypertrophy}

Cardiac hypertrophy is an adaptive process that improves ventricular function in the face of a growing workload. Although beneficial in the short-term, persistent hypertrophy is detrimental to cardiac efficiency. Reduced contractility and an increased risk of heart failure are associated with sustained cardiac hypertrophy. The adult myocardial cell is a fully differentiated type of cell. Consequently, cardiac hypertrophy results in an increase in the size of cells as opposed to an increase in cell number. Hypertrophic responses are not limited to external stressors, such as increased afterload, but can also be congenital. For example, in Familial Hypertrophic Cardiomyopathy (FHC), ventricular hypertrophy has been associated with mutations that encode sarcomeric proteins (14). More than 100 different models have been developed to study the molecular changes present in hypertrophic cells, but the molecular mechanisms that differentiate its adaptive and pathologic effects are not yet fully understood.

One common use of murine models for hypertrophy has been in the investigation of contractile protein abnormalities. Mutations in myosin, actin, and many other filament proteins have been genetically manipulated to model hypertrophy. In most of these cases, the homozygous knockout mice have fatal complications before adulthood, whereas heterozygous knockouts or expression of specific mutant forms produce hypertrophic symptoms. Alpha cardiac myosin heavy chain point mutation $\mathrm{R} 403 \mathrm{Q}$ has produced a murine phenotype that closely resembles human FHC (15). Cytoskeletal proteins have also been identified as proteins of consequence in cardiac hypertrophy because of the involvement of mutant isoforms in genetically based forms of dilated cardiomyopathy. In particular, muscle-LIM protein-deficient mice express a phenotype that approximates heart failure and cardiomyopathy in humans (16). Cell surface receptors and the complex signal cascades that are activated are responsible for differentiating the cellular responses to external stimuli. Many receptors and signaling molecules play critical roles in mediating the hypertrophic response in cardiomyocytes. A large number of transgenic and knockout models have been created to elucidate these signaling pathways whose activation leads to eventual heart failure. An example of this is the $\beta$-adrenergic receptor $(\beta-A R)$ signaling pathway, which serves as the interface between the sympathetic nervous system and the cardiovascular system. This pathway is dramatically altered in a number of cardiovascular diseases, including hypertrophy, and it is one of the best characterized signaling pathways in the heart (17). Genetically manipulated models exist for many of the member molecules of this pathway, such as $\beta$ adrenergic receptor kinase-1 (18). One potential therapeutic approach for heart failure that has been revealed from this research is the systemic overexpression of the $\beta 2$-AR (19). Murine models demonstrated that overexpression of $\beta 2$-ARs produces enhanced contractility.

\section{Myocardial Ischemia, Reperfusion and Angiogenesis}

Myocardial ischemia is the principal cause of mortality in Western countries. Temporary coronary ischemia and reperfusion in humans who suffered myocardial infarction followed by thrombolytic therapy or cardiac catheterization can be mimicked in mouse models. In these models, the left anterior descending coronary artery is occluded for $30 \mathrm{~min}$ followed by reperfusion of blood through the previously occluded coronary artery bed (20). Prompt reperfusion of ischemic myocardium is essential for restoring heart function. However, this return of blood flow can paradoxically augment the destruction of reversibly damaged myocytes, thus exacerbating myocardial dysfunction and infarction (21). Murine models of ischemia and reperfusion help to understand the molecular mechanisms of myocardial response to ischemia in humans and enhance treatment options. In these mice, the area at risk, infarct size, and cardiac function are determined by ultrasound biomicroscopy and histopathologic techniques. Understanding angiogenesis, the sprouting of new blood vessels from existing ones, is important because the extent of myocardial infarction after coronary artery occlusion is substantially influenced by collateral circulation. Also, therapeutic angiogenesis may be the only treatment option in certain pathologic conditions (22). In models of angiogenesis, limb ischemia is induced by permanent ligation of the femoral artery and collateral vessel formation is monitored by microangiography.

\section{TECHNOLOGY}

\section{Genetic Technology}

Transgenesis, the transfer of cloned genetic material, is used to introduce a gene of interest into the animal 
model for study. Expression is restricted to a specific tissue by fusing the candidate gene to a cardiac-specific promoter in the transgenic construct. To produce a stable genetic modification, the construct is injected into the pronucleus of a one-cell embryo. The embryo is then implanted into a pseudopregnant female mouse. Mouse pups are genotyped and the transgenic mice, the founders, are mated to produce the stable line (6). Transgenesis is typically used to induce overexpression of transcriptional levels of the candidate gene. However, neither the copy number nor the point of insertion can be controlled, and both the transgene and the native gene are expressed. Consequently, it is necessary for the transgene expression to be dominant over its homologue in order to produce a phenotype. Furthermore, DNA insertion can result in mutagenic effects in the flanking DNA, complicating any resultant phenotype. Hence, several independent founders are generated to check the levels and sites of transgene expression and compare the phenotypes. Transgenesis is typically used to investigate endogenous protein function and signal transduction pathways.

Alternatively, gene-targeting procedures are used to disable or 'knock out' a candidate gene (23). A construct is designed that has two regions of homology to the target gene, flanking a selectable marker. These markers are bacterial genes that offer resistance to neomycin, hygromycin, or puromycin, which will disrupt the segments of DNA that are either essential for transcription and/or translation. Recombination between the flanking sequences of homology in the targeting construct and the genomic locus replaces the host gene sequence with the selectable marker. The cells that have the selectable marker integrated into their genome are chosen by means of molecular screening techniques prior to being microinjected into blastocysts and implanted into pseudopregnant mice. The heterozygous mice that are produced are mated together to generate homozygous knockout mice, which lack a functional copy of the candidate gene. Genetargeting is sometimes used to generate mice with a mutation in, rather than inactivation of, the candidate gene. In these cases, a construct identical to the endogenous DNA (other than the desired mutation) is made. Compared to transgenesis, gene-targeting is an elegant system because it can control both the number of copies as well as the point of insertion. More accurate cardiac-phenotype modeling is achieved via the absence of insertional mutagenic effects, the complete elimination of the endogenous candidate gene, and its expression in specific cells and tissues. Furthermore, in cases where gene-targeting is used for expressing mutated genes, the mutated gene will be expressed under the same transcriptional patterns as the gene of interest. The experimental advantages of genetargeting are offset to some degree by its relative laborintensiveness; transgenesis can yield a stable mouseline in a matter of months, whereas a gene-targeting line can take years to come to fruition.

\section{MRI (Magnetic Resonance Imaging) in Murine Cardiac Research}

MRI is a well-established diagnostic technique that uses a magnetic field to generate high-contrast images of structures inside the human body on the basis of proton magnetic fields. Traditionally, MRI has been used for detecting tumors and structural abnormalities in soft-tissue organs such as the brain, spinal cord, heart, and eye. Information from MRI can help to determine when a stroke occurred, support a diagnosis of multiple sclerosis, and identify problems of the brain and spinal cord, which might not be detectable with other imaging techniques. MRI can also be used for such subtle applications as distinguishing between a tumor and benign fibrocystic disease. Recently, MRI has been adapted for use in murine cardiac research. MRI is ideal for these studies because it can generate high-resolution images across many time-points. In the past, studies about the physical changes involved in cardiovascular disease (such as hypertrophy, arterial stenosis and remodeling, or atherosclerotic lesions) were constrained by the need to maintain multiple experimental groups with a large number of mice killed at different time-points. Currently, cardiac MRI is an effective detector of hypertrophy and arterial remodeling/stenosis (24). The technique can also effectively identify and characterize atherosclerotic lesion burden in live mouse subjects. The clinical and laboratory applications of cardiac MRI are advancing hand-in-hand. MRI is already used for imaging the aorta and carotid arteries in human patients. The viability of MRI characterization of human atherosclerotic plaque has already been demonstrated (25). Once fully adapted to murine cardiology, MRI could yield information about the progression of cardiovascular disease by characterizing the size, scope, and biochemical complexion of atherosclerotic plaque buildup in blood vessels.

\section{Echocardiogram}

Echocardiograms, among the most widely applicable technologies in clinical cardiology, have been adapted for noninvasive physiologic studies in mice. Echocardiograms are particularly useful because they can provide a generalized picture of the structure and function of the heart throughout the life of the specimen. Motion mode, or M-mode, analysis of echocardiography is the procedure most often used in 
mouse studies. The M-mode analysis is optimized for higher sampling frequency and smaller heart size to accommodate the mouse's diminutive size and resultant rapid heart rate (26). Typically the specimen is weighed and anesthetized before the echocardiogram is performed. Measurements for the anterior and inferior wall-thickness, left-ventricular end-diastolic dimension, and left-ventricular and cardiac mass can all be measured to a high degree of precision by echocardiography (27). Other cardiac endpoints such as contractility, left-ventricular dilation, and fractional shortening can also be determined from images produced by echocardiograms (26). Factors such as heart rate can be controlled through the use of different anesthesia protocols. The data from these procedures provide a detailed and holistic view of cardiac function; data comparisons from normal and genetically altered models can be used to understand the effect of the genetic alteration in the context of whole heart function.

\section{Ultrasound Biomicroscopy}

Similar to the ultrasound scanners in hospitals, ultrasound biomicroscopes can evaluate cardiac structure and function in mice. This system, in addition to using M-Mode imaging for echocardiogram, uses two-dimensional B-Mode for anatomical imaging, and pulsed Doppler processing to measure blood flow in the mouse cardiovascular system. This non-invasive procedure would allow researchers to observe a number of changes over time, such as 1) during the development phase, changes in the ventricular diastolic filling patterns that are due to genetic manipulation, 2) disease progression, and 3) real-time responses to experimental treatment in a single mouse. This would enable investigators to screen large numbers of mice as models of human disease.

\section{NEW DIRECTIONS}

Since the arrival of molecular genetics technology, transgenic and knockout mice models have proven to be elegant and powerful experimental models for answering questions about the function of individual molecules and genes. However, recent evidence suggests that researchers should exercise caution when drawing conclusions about the role of molecules in pathogenesis. From the beginning, researchers observed that the genetic background of specific strains could potentially affect the severity of the phenotype that is expressed, as the atherosclerosis and hyperlipidemia apoE -/- phenotypes were only fully expressed in the C57BL/6 background. Researchers who use the apoE deficient mice for additional genetic manipulation must make the effort to backcross with C57BL/6 multiple times or risk skewed results (28).
Precautions must also be taken to prevent positiondependent non-specific events caused by random insertional mutations during transgenesis. Transgenic expression of presumably ectopic proteins can also compromise results, as it has been shown that transgenic expression of GFP, normally considered nonreactive, can cause dilated cardiomyopathy (29). Perhaps even more worrying are the consequences of gene redundancy and compensatory responses (30). Evidence indicates that the molecular events under investigation are part of immensely complex biological processes governed by multiple genetic and molecular influences. Crudely knocking out or overexpressing a protein may activate normally dormant compensatory mechanisms. In such a scenario, the phenotype expressed would not be indicative of a loss of actual function. This sort of difficulty will be eliminated with the emergence of more refined genetic technologies that will allow for more cell type-specific and timegoverned genetic manipulation.

There are several collaborative projects underway to improve the resources available to researchers using genetically manipulated models. Two of the most ambitious are the Mouse Phenome Project undertaken by Jackson Laboratories (Bar Harbor, Maine; www.jax.org) and a Complex Trait initiative begun by Complex Trait Organization (Memphis, Tennessee; www.complextrait.org). The Mouse Phenome Project is an attempt to create a public database of detailed phenotypic data on the most common inbred strains. They also hope to generate new lines that closely represent human disease through directed breeding based on the natural variation among extant mouse strains and chemically induced, whole-genome mutagenized mice. The creation of the database and the generation of new models will allow researchers to make informed decisions about what background to use for genetic manipulation when making new models. The information will also help scientists sort out straindependent effects when interpreting data. Geneticists at the Complex Trait Organization want to locate genes involved in complex traits by creating 1000 new lines of "recombinant inbred" mice. Each line would be a combination of eight existing strains; the scientists would outcross the mice for four generations and then breed brother-sister pairs for 20 generations to create inbred lines. Researchers hope the new lines will be able to reveal the genes that interact to produce complex, non-Mendelian traits and represent the variation in natural mouse populations. The emergence of functional genomics presents a great new opportunity for research involving genetically manipulated mouse models. New technologies such as gene microarrays let researchers look at the activation of thousands of genes 
at any given time. This is especially advantageous in the study of complex polygenetic diseases such as cardiovascular disease. As this and other functional genomic technologies mature, researchers will have powerful new tools to examine complex genetic interactions.

\section{ACKNOWLEDGEMENT}

I am grateful to Drs. Sathyamangla V. Naga Prasad and Howard Rockman at Duke University, and Drs. Cam Patterson and Marschall S. Runge at University of North Carolina, Chapel Hill, for their critical reading of the manuscript.

\section{REFERENCES}

1. Doevendans PA, J. Daemen M, de Muinck ED and Smits JF. Cardiovascular phenotyping in mice. Cardiovascular Research. 39: 34-49; 1998.

2. Piedrahita JA, Zhang SH, Hagaman JR, Oliver PM, Maeda N. Generation of mice carrying a mutant apolipoprotein $\mathrm{E}$ gene inactivated by gene targeting in embryonic stem cells. Proc. Natl. Acad. Sci. U S A. 89: 4471-44755; 1992.

3. Zhang SH, Reddick RL, Piedrahita JA, Maeda N. Spontaneous hypercholesterolemia and arterial lesions in mice lacking apolipoprotein E. Science. 258: 468-471; 1992

4. Navab M, Berliner JA, Watson AD et al. The Yin and Yang of oxidation in the development of the fatty streak. A review based on the 1994 George Lyman Duff Memorial Lecture. Arterioscler. Thromb. Vasc. Biol. 16:831-842; 1996.

5. Heistad DD. Unstable coronary-artery plaques. N. Engl. J. Med.. 349:2285-2287; 2003.

6. Daugherty A. Mouse models of atherosclerosis. Am. J. Med. Sci. 323: 3-10: 2002.

7. Van den Maagdenberg AMJM, Frants RR et al. Transgenic mice carrying the apoliprotein E3-Leiden gene exhibit hyperlipoproteinemia. J. Biol. Chem. 268:10540-10546;1993.

8. Fazio S, Linton MF. Mouse models of hyperlipidemia and atherosclerosis. Front. Biosci. 6: D515-D5225; 2001.

9. Ishibashi S, Brown MS, Goldstein JL, Gerard RD, Hammer RE, Herz J. Hypercholesterolemia in density lipoprotein receptor knockout mice and its reversal by adenovirus-mediated gene delivery. J. Clin. Invest. 92: 883-893; 1993.

10. Sullivan PM, Mezdour H, Quarfordt SH, Maeda N. Type III hyperlipoproteinemia and spontaneous atherosclerosis in mice resulting from gene replacement of mouse Apoe with human Apoe*2. J. Clin. Invest. 102: 130-135; 1998.

11. Carmeliet P, Moons L, Stassen JM, et al. Vascular wound healing and neointima formation induced by perivascular electric injury in mice. Am. J. Pathol. 150: 761-776, 1997.

12. Chong PH, Cheng JW. Early experiences and clinical implications of restenosis and drug-eluting stents: Part 2. Ann Pharmacother. 38:845-852; 2004.

13. Lindner V, Fingerle J, Reidy MA. Mouse model of arterial injury. Circ. Res. 73: 792-796: 1993.
14. Thierfelder L, Watkins H, MacRae C, et al. Alpha-tropomyosin and cardiac troponin $\mathrm{T}$ mutations cause familial hypertrophic cardiomyopathy: a disease of the sarcomere. Cell. 77:701-712; 1994.

15. Geisterfer-Lowrance AA, Christe M, Conner DA, et al. A mouse model of familial hypertrophic cardiomyopathy. Science. 272:731-734; 1996.

16. Pashmforoush M, Pomies P, Peterson KL, et al. Adult mice deficient in actinin-associated LIM-domain protein reveal a developmental pathway for right ventricular cardiomyopathy. Nat. Med. 7: 591-597; 2001

17. Choi D-J, Koch WJ, Hunter JJ, Rockman HA. Mechanism for ßadrenergic receptor desensitization in cardiac hypertrophy is increased B-adrenergic receptor kinase. J. Biol. Chem. 272:17223-17229; 1997.

18. Koch WJ, Rockman HA, Samama P, et al. Cardiac function in mice overexpressing the beta-adrenergic receptor kinase or a beta ARK inhibitor. Science. 268:1350-1353, 1995.

19. Tevaearai HT, Eckhart AD, Walton GB, Keys JR, Wilson K, Koch WJ. Myocardial gene transfer and overexpression of beta2-adrenergic receptors potentiates the functional recovery of unloaded failing hearts. Circulation. 106:124-129, 2002.

20. Metzler B, Mair J, Lercher A, et al. Mouse model of myocardial remodelling after ischemia: role of intercellular adhesion molecule-1. Cardiovasc. Res. 49:399-407; 2001.

21. Maxwell SR, Lip GY. Reperfusion injury: a review of the pathophysiology, clinical manifestations and therapeutic options. Int. J. Cardiol. 58:95-117; 1997.

22. Emanueli C, Madeddu P. Angiogenesis gene therapy to rescue ischaemic tissues: achievements and future directions. $\mathrm{Br} \mathrm{J}$ Pharmacol. 133:951-958; 2001.

23. Doetschman T, Maeda N, Smithies O. Targeted mutation of the Hprt gene in mouse embryonic stem cells. Proc. Natl. Acad. Sci. U S A. 85:8583-8587; 1988.

24. Fayad ZA, Fallon JT, Shinnar M, et al. Noninvasive in vivo high-resolution magnetic resonance imaging of atherosclerotic lesions in genetically engineered mice. Circulation. 98: 15411547; 1998.

25. Pohost GM and Fuisz AR. From the microscope to the clinic : MR assessment of atherosclerotic plaque. Circulation. 98: 14771478; 1998.

26. James JF, Hewett TE and Robbins J. Cardiac physiology in transgenic mice. Circ Res. 82: 407-415; 1998.

27. Hoit BD, Khoury SF, Kranias EG, Ball N and Walsh RA. In vivo echocardiographic detection of enhanced left ventricular function in gene-targeted mice with phospholamban deficiency. Circ Res. 77: 632-637; 1995.

28. Knowles JW and Maeda N. Genetic modifiers of atherosclerosis in mice. Arterioscler Thromb Vasc Biol. 20: 2336-2345; 2000

29. Huang W-Y, Aramburu J, Douglas PS and Izumo S. Transgenic expression of green fluorescence protein can cause dilated cardiomyopathy. Nature Medicine. 6: 482-483; 2000.

30. Chu G, Haghighi $\mathrm{K}$ and Kranias EG. From mouse to man: Understanding heart failure through genetically altered mouse models. Journal of Cardiac Failure. 8: S432-S449; 2002.

\footnotetext{
Aasakiran Madamanchi is a third year undergraduate student at Duke University. His area of interest includes research in the molecular mechanisms of heart failure. He has worked in the Laboratory of Dr. Howard Rockman in the Cell Biology Department at Duke University for the last several years and he hopes to attend medical school following the completion of his Bachelors of Science in Biology degree.
} 